neofilolog

Czasopismo Polskiego Towarzystwa Neofilologicznego

ISSN 1429-2173, eISSN 2545-3971, 2020, NR 55/2, 173-194

http://dx.doi.org/10.14746/n.2020.55.2.2

http://poltowneo.org/

Radosław Kucharczyk

Uniwersytet Warszawski https://orcid.org/0000-0002-1861-1358

r.kucharczyk@uw.edu.pl

Krystyna Szymankiewicz

Uniwersytet Warszawski https://orcid.org/0000-0002-8598-3418

k.szymankiewicz@uw.edu.pl

\title{
Les représentations de la langue française chez des élèves polonophones. Quel impact sur le choix de la langue étrangère à l'école ? ${ }^{1}$
}

\begin{abstract}
Polish speaking pupils' representations of French. What impact does this have on the choice of a foreign language at school?
\end{abstract}

The aim of our article is to present a study of representations of the French language among Polish high school students. Given the small percentage of pupils learning French as Foreign Language (FFL) in Poland compared to the European average $(33.17 \%$ of middle school students and $23.0 \%$ of high school students in Europe against $3.3 \%$ of middle school students and $10.8 \%$ of high school students in Poland (Eurydice 2017, GUS 2018), we wondered about the role that impressions relating to French can play in the choice or rejection of FFL by young Poles. Before presenting our research results relating to this problem, we give a brief description of the Polish sociolinguistic and educational context, then we approach the notion of representation / image of languages.

\footnotetext{
${ }^{1}$ L'article présente la première étape d'une étude plus vaste, que nous relatons dans un autre article intitulé « Uwarunkowania wyboru nauki języka francuskiego przez polskich licealistów » (paru dans Acta Neofilologica no1 /2020) où sont comparés les motifs du choix de l'apprentissage du FLE chez les lycéens de Varsovie et chez les lycéens de province.
} 
Next, we move to the description of the methodological framework of the empirical study that we conducted with Polish-speaking high school students (learners and non-learners in FFL) before presenting the discussion of the results. On the one hand, we examined the impressions relating to French (defined as linguistic, aesthetic, cultural, social and political phenomenon), on the other representations relating to its usefulness.

Keywords: French, Poland, learning, representations, research

Słowa kluczowe: język francuski, Polska, uczenie się, reprezentacje społeczne, badania

\section{Introduction}

Suite à la politique linguistique du Conseil de l'Europe promouvant le plurilinguisme, les systèmes scolaires des pays européens ont mis en œuvre l'enseignement d'au moins deux langues étrangères dont la première est enseignée à partir de l'âge de 5 ans et la deuxième à partir de l'âge de 13 ans $^{2}$. L'apprentissage des deux langues continue jusqu'à la fin du secondaire. Dans la plupart des cas, c'est l'anglais qui jouit de la position de première langue étrangère enseignée, en raison de son statut indéniable de langue internationale. En ce qui concerne la deuxième langue étrangère, son choix se fait dans le cadre de l'offre préparée par l'établissement scolaire et peut être influencé par les représentations que possèdent les élèves des langues et de leur apprentissage.

Dans notre article, nous voudrions présenter une étude des représentations de la langue française chez des lycéens polonais. Étant donné le faible pourcentage d'élèves apprenant le Français Langue Etrangère (désormais : FLE) en Pologne par rapport à la moyenne européenne $(33,17 \%$ de collégiens et $23,0 \%$ de lycéens en Europe contre $3,3 \%$ de collégiens et $10,8 \%$ de lycéens en Pologne ( $c f$. Eurydice 2017, GUS 2018)), nous nous sommes interrogés sur le rôle que peuvent jouer les représentations relatives au français dans le choix ou le rejet du FLE par les jeunes Polonais. Avant d'exposer nos résultats de recherche relatifs à ce problème, nous procédons à une brève description du contexte sociolinguistique et éducatif polonais, puis nous abordons la notion de représentation/image des langues. Ensuite, nous passons à la description du cadre méthodologique de l'étude empirique que nous avons menée auprès des lycéens polonophones

${ }^{2}$ Ce qui correspond actuellement à la $7^{\mathrm{e}}$ classe de l'école primaire polonaise ; avant la réforme de 2018 supprimant les collèges, l'enseignement de la deuxième langue étrangère commençait durant la $1^{\text {ère }}$ classe du collège. 
Les représentations de la langue française chez des élèves polonophones...

(apprenants et non-apprenants en FLE) pour présenter enfin la discussion des résultats : d'une part nous avons examiné les représentations relatives à la langue française (définie en tant que phénomène linguistique, esthétique, culturel, social et politique), de l'autre des représentations relatives à son utilité.

\section{Le contexte sociolinguistique et éducatif polonais}

La Pologne reste un pays monolingue, dans le sens où il y existe une seule langue officielle (le polonais) et que le nombre d'étrangers (y compris les immigrés) reste relativement peu élevé. II s'ensuit donc que le contact que les élèves polonais ont avec les langues étrangères se fait avant tout à l'école et grâce aux médias. Face au déferlement de l'anglais et de la culture anglo-saxonne dans les médias et sur Internet, la présence du français semble marginale.

Ensuite, en Pologne, le français langue étrangère est enseigné en tant que deuxième langue étrangère au choix, l'anglais étant la première langue étrangère obligatoire pour tous les élèves. II en résulte un écart énorme entre le nombre d'heures d'enseignement de l'une et de l'autre langue : au total, dès le début du primaire jusqu'à la fin du secondaire, les écoliers peuvent assister à 990 heures de cours d'anglais contre seulement 360 heures de cours de deuxième langue étrangère (Ministerstwo Edukacji Narodowej, 2016 : 2). Par conséquent, l'apprentissage du français en tant que deuxième langue étrangère est souvent perçu par les lycéens comme futile, puisque de toute façon, vu sa faible intensité et le manque d'exposition au français en dehors du cadre scolaire, il ne peut pas mener à une bonne maîtrise de la langue (cf. Błaszkiewicz, 2017).

Dans ce contexte, il est à noter que le nombre d'élèves qui choisissent d'étudier le français baisse d'année en année : $3,3 \%$ d'élèves (tout type d'école compris) en 2005/2006, 2,9 \% en 2010/2011, 2,4 \% en 2015/2016 et 2,3 \% en 2017/2018 (GUS, 2018). Ainsi, le français se voit concurrencé non seulement par l'anglais, mais aussi par d'autres langues dont l'enseignement est offert par l'école, à savoir l'allemand (36,4 \% d'élèves en $2017 / 2018)$ et le russe $(3,6 \%)$ ou - plus récemment - l'espagnol (2,5\%) selon les données du GUS (2018:63).

Compte tenu du statut formel du français dans le système scolaire polonais, nous avons trouvé utile, pour comprendre les raisons de la faible popularité du FLE, d'analyser son statut informel (Dabène, 1997 : 20) qui se cristallise dans les représentations des langues des apprenants polonais.

\section{Les représentations des langues}

En didactique des langues étrangères, l'intérêt pour les représentations ou images des langues se fait remarquer depuis plusieurs années, notamment en 
rapport à des aspects tels que : le développement de la compétence interculturelle, la construction identitaire du sujet, le choix de la langue que l'on veut apprendre, les stratégies mises en œuvre lors de son apprentissage ainsi que les résultats obtenus ( $c f$. Zarate, 1993 ; Dabène, 1997 ; Castellotti \& Moore, 2002, Castellotti, 2017 ; Defays, 2018). Rappelons que le terme de représentations ou images des langues se réfère à la notion de représentations sociales, faisant, quant à elle, l'objet d'investigations dans plusieurs domaines des sciences humaines, comme la psychologie sociale ( $c f$. Moscovici, 1961, 1976), la sociologie ( $c f$. Jodelet 1993) ou l'anthropologie. L'une des définitions les plus souvent citées est celle de Denise Jodelet qui met en relief le caractère cognitif, social et partagé des représentations sociales ainsi que leur fonction de (re)construction et de clarification de la réalité. Ainsi, les représentations sociales constituent " (...) une forme de connaissance, socialement élaborée et partagée, ayant une visée pratique et concourant à la construction d'une réalité commune à un ensemble social » (Jodelet, 1993 : 36).

Il s'agit d'un "système d'interprétation " fort complexe, car comportant des éléments cognitifs, idéologiques, affectifs, normatifs, des attitudes, opinions et valeurs (ibidem). C'est de la co-action de tous ces éléments que se dégage le sens attribué à un objet de la réalité. Ce qui paraît important pour notre propos, c'est que les représentations sociales peuvent déterminer les conduites et les comportements des individus envers un objet donné. Une autre caractéristique des représentations sociales consiste dans le fait qu'elles sont modifiables et qu'elles peuvent évoluer aussi bien sur le plan collectif que sur le plan individuel.

Utilisé en référence aux langues, le concept de représentations est défini comme " les images et les conceptions que les acteurs sociaux se font d'une langue, de ce que sont ses normes, ses caractéristiques, son statut au regard d'autres langues " (Moore, 2001 : 9). Danièle Moore souligne l'existence d'une forte affinité entre les images des langues, les attitudes et les stéréotypes culturels (ibidem). L'attitude marque une orientation individuelle en tant que « disposition à réagir de manière favorable ou non à une classe d'objet " (ibidem : 13). En revanche, les stéréotypes ont un caractère collectif, car ils constituent plutôt " une forme spécifique de verbalisation des attitudes, caractérisée par l'accord des membres de l'endo-groupe autour de certains traits saillants, adoptés comme valides et discriminants " (Tajfel, $1981^{3}$, cité par Moore, $2001: 14)$. Il en résulte que les comportements des individus peuvent être déterminés par leurs attitudes et les stéréotypes qu'ils partagent. À

${ }^{3}$ Tajfel H. (1981), Human groups and social categories: Studies in social psychology. Cambridge: Cambridge University Press, p. 115. 
titre d'exemple, les stéréotypes attachés à la langue française par les étrangers la présentent souvent comme " une langue élégante, raffinée, subtile et par conséquent difficile, avec des règles complexes, des exceptions nombreuses " (Defays, 2018 : 37). Cette image d'une langue difficile, renforcée par le purisme des Français, aurait pour effet de décourager de potentiels apprenants en FLE et de compromettre la diffusion internationale du français (ibidem : 38 ).

Ainsi, le choix de la langue étrangère que l'apprenant veut apprendre dépend en grande partie des représentations - positives ou négatives - qu'il se fait de cette langue, de ses natifs et de la culture qui y liée (Defays, 2018 : 37).

Afin de soumettre les images des langues à une analyse de contenu, il nous semble utile de nous référer au classement de critères d'appréciation sur lesquels se fondent ces représentations, proposé par Louise Dabène (Dabène, 1997 : 21-22) :

- le critère économique (l'utilité de la langue dans les différents domaines de la vie) : à quel point la langue assure-t-elle l'accès au monde du travail ?

- le critère social (le prestige de la langue) : quel est le niveau social des locuteurs de la langue ? Quelles sont les possibilités de promotion ou d'ascension sociale liées à la connaissance de la langue ?

- le critère culturel : peut-on associer la langue à une richesse culturelle (littérature, passé historique glorieux, etc.) ?

- le critère épistémique : quelles sont les exigences cognitives liées à l'apprentissage de la langue ? (facile/difficile)

- le critère affectif : quels préjugés (favorables/défavorables) associe-ton à la langue ? (p. ex. sous l'influence de l'histoire du pays ou/et de l'histoire personnelle).

À cette liste nous avons ajouté, suite à Defays (2018 : 37), un critère supplémentaire, à savoir celui de la beauté/laideur de la langue, que nous avons appelé " esthétique ". Les critères cités ci-dessus nous ont servi de points de repère dans notre étude dont le cadre méthodologique et les résultats seront présentés dans les sections qui suivent.

\section{Le cadre méthodologique de la recherche empirique}

\subsection{Les questions et les hypothèses de recherche}

Pour réaliser notre étude empirique, nous nous sommes posé des questions de recherche suivantes : 
1. Quelles sont les représentations relatives à la langue française fonctionnant chez les jeunes Polonais ?

2. Ya-t-il des différences significatives entre les représentations relatives à la langue française fonctionnant chez les apprenants en FLE et les non-apprenants en FLE ?

3. Quels sont les critères d'appréciation du français qui peuvent jouer un rôle positif dans le choix de l'apprentissage de cette langue en tant que deuxième langue étrangère au lycée ?

4. Quels sont les critères d'appréciation du français qui peuvent décourager les lycéens polonais de choisir l'apprentissage de cette langue en tant que deuxième langue étrangère ?

Une telle formulation des questions de recherche nous a permis d'émettre les hypothèses suivantes qui se fondent sur nos observations ainsi que sur les résultats d'autres recherches dans la même veine (Pudo, 2016 ; Ziółkowski, 2004) :

1. Les représentations relatives à la langue française fonctionnant chez les jeunes Polonais sont plutôt stéréotypées : le français est perçu comme une langue liée à la culture, une langue belle mais difficile à apprendre, moyennement utile sur le marché du travail.

2. Les apprenants en FLE perçoivent la langue française de manière moins stéréotypée que les non-apprenants : leurs représentations sont plus " réalistes » et plus positives. Les apprenants en FLE perçoivent le français comme une langue plus utile que les non-apprenants en FLE.

3. Les critères qui peuvent influer positivement sur le choix du français en tant que deuxième langue étrangère à apprendre au lycée sont avant tout d'ordre pragmatique (l'utilité de la langue sur le marché du travail, dans les institutions de l'Union européenne) et esthétique (la beauté de la langue).

4. Les critères d'appréciation qui peuvent décourager du choix du français en tant que deuxième langue étrangère à apprendre au lycée sont avant tout liés à la perception de la difficulté de la langue française et à la faible présence du français en Pologne, entraînant la conviction d'une utilité modérée de cette langue.

\subsection{L'outil de la recherche}

Nous avons élaboré un questionnaire d'enquête se composant de 4 parties. Toutes les questions étaient fermées : vu le nombre considérable de participants à l'étude (voir 4.3.), cette décision nous a semblé la plus réaliste. Le tableau qui suit présente les caractéristiques de notre questionnaire : 
Les représentations de la langue française chez des élèves polonophones...

\begin{tabular}{|c|c|c|c|}
\hline $\begin{array}{c}\text { partie du } \\
\text { questionnaire }\end{array}$ & groupe cible & questions & objectif(s) \\
\hline I. & tous les apprenants & -- & $\begin{array}{l}\text { recueillir des données signalétiques (sexe, } \\
\text { classe, langues apprises à l'école primaire } \\
\text { et au collège) }\end{array}$ \\
\hline \multirow[t]{3}{*}{ III. } & \multirow[t]{3}{*}{ tous les apprenants } & I. & $\begin{array}{l}\text { examiner les représentations des élèves, } \\
\text { relatives à la langue française perçue en } \\
\text { tant que phénomène }{ }^{4} \text { : } \\
\text { - linguistique (items : I.19, I.20, I.22, I.23) } \\
\text { - esthétique (items : I.3, I.10, I.11, I.12, } \\
\text { I.17, I.21, I.24) } \\
\text { - culturel (items : I.1, I.2, I.4, I.5, I.6, I.7, I.9) } \\
\text { - social (items : I.13, I.14, I.15, I.16) } \\
\text { - politique (items : I.8, I.8, I.25, I.26) }\end{array}$ \\
\hline & & III. & $\begin{array}{l}\text { examiner les représentations sociales relatives } \\
\text { à l'utilité de connaître la langue française }\end{array}$ \\
\hline & & III. & $\begin{array}{l}\text { examiner le contact des élèves interrogés } \\
\text { avec la langue française }\end{array}$ \\
\hline \multirow[t]{4}{*}{ III. } & \multirow[t]{4}{*}{ les apprenants en FLE } & IV. & $\begin{array}{l}\text { examiner les motifs de l'apprentissage de } \\
\text { la langue française }\end{array}$ \\
\hline & & V. & $\begin{array}{l}\text { estimer le degré de facilité de l'apprentis- } \\
\text { sage du français }\end{array}$ \\
\hline & & VI. & $\begin{array}{l}\text { s'informer sur le nombre de cours de fran- } \\
\text { çais par semaine }\end{array}$ \\
\hline & & VII. & $\begin{array}{l}\text { s'informer si les apprenants auraient choisi } \\
\text { le français s'ils avaient dû choisir encore } \\
\text { une fois les langues étrangères au lycée }\end{array}$ \\
\hline IV. & $\begin{array}{l}\text { les non-apprenants en } \\
\text { FLE }\end{array}$ & VIII. & $\begin{array}{l}\text { examiner les motifs du non-apprentissage } \\
\text { du français }\end{array}$ \\
\hline
\end{tabular}

Tableau 1 : Caractéristique du questionnaire de l'enquête.

\subsection{L'échantillon participant à l'étude}

Nous avons mené notre étude empirique auprès d'élèves polonophones venant de 3 lycées différents de Varsovie. Notre choix n'a pas été aléatoire. D'abord, les apprenants âgés de 16 à 19 ans ${ }^{5}$ ont déjà une certaine expérience relative aux langues et à la connaissance du monde, ce qui leur permet de s'exprimer de façon consciente sur les représentations. De plus, tous les élèves

\footnotetext{
${ }^{4}$ Pour élaborer la cafétéria d'items relatifs aux représentations de la langue française, nous avons lancé une discussion libre sur un réseau social (Terminez la phrase " La langue française me fait penser à ... »), ce qui nous a permis d'intégrer, dans le questionnaire de l'enquête, les réponses les plus pertinentes.

${ }^{5} C^{\prime}$ est l'âge où les apprenants polonophones fréquentent l'école secondaire, le lycée en l'occurrence.
} 
fréquentent un lycée à Varsovie où l'exposition à la langue française est relativement élevée par rapport à la province polonaise. Enfin, le français est enseigné dans toutes les écoles où nous avons mené notre étude, ce qui permet de supposer que le choix de l'école, de même que le choix des langues à apprendre, a été un choix conscient des élèves. Force est quand même de constater que l'une des écoles était un établissement comportant des sections bilingues francophones où tous les élèves apprennent le français en tant que première langue étrangère obligatoire (dans les classes bilingues et non bilingues). Au total nous avons interrogé 556 apprenants ( $N=556), y$ compris 320 apprenants en FLE $\left(\mathrm{N}_{1}=320\right)$ et 236 non-apprenants en FLE $\left(\mathrm{N}_{2}=236\right)$. Leurs caractéristiques seront présentées dans les sections qui suivent.

\subsubsection{Les apprenants en FLE}

\section{La caractéristique générale}

Les tableaux qui suivent présentent les caractéristiques générales des apprenants en FLE : sexe, classe, nombre d'heures de cours de français par semaine, langues apprises au primaire et au collège.

\begin{tabular}{|c|c|c|c|}
\hline \multirow{2}{*}{$N_{1}=320$} & femme & homme & $\begin{array}{c}\text { manque } \\
\text { d'informations }\end{array}$ \\
\cline { 2 - 4 } & 253 & 67 & 0 \\
$(79 \%)$ & $(21 \%)$ & $(0 \%)$ \\
\hline
\end{tabular}

Tableau 2 : Les apprenants en FLE : sexe.

\begin{tabular}{|c|c|c|c|c|}
\hline \multirow{3}{*}{$N_{1}=320$} & $\mathbf{1}^{\text {ère }}$ & $\mathbf{2}^{\mathbf{e}}$ & $\mathbf{3}^{\mathbf{e}}$ (terminale) & $\begin{array}{c}\text { manque } \\
\text { d'informations }\end{array}$ \\
\cline { 2 - 5 } & $\begin{array}{c}181 \\
(57 \%)\end{array}$ & $\begin{array}{c}108 \\
(33 \%)\end{array}$ & $\begin{array}{c}31 \\
(10 \%)\end{array}$ & $\begin{array}{c}0 \\
(0 \%)\end{array}$ \\
\hline
\end{tabular}

Tableau 3 : Les apprenants en FLE : classe.

\begin{tabular}{|l|c|c|c|c|c|c|}
\hline & $\mathbf{1}$ heure & $\mathbf{2}$ heures & $\mathbf{3}$ heures & $\mathbf{4}$ heures & $\begin{array}{c}\mathbf{5} \text { heures et } \\
\text { plus }\end{array}$ & $\begin{array}{c}\text { manque } \\
\text { d'informations }\end{array}$ \\
\hline $\mathbf{N}_{\mathbf{1}}=\mathbf{3 2 0}$ & 2 & 23 & 161 & 8 & 124 & 0 \\
\hline$\%$ & $1 \%$ & $7 \%$ & $50 \%$ & $3 \%$ & $39 \%$ & $0 \%$ \\
\hline
\end{tabular}

Tableau 4 : Nombre d'heures de cours de français par semaine.

\begin{tabular}{|c|c|c|c|c|c|c|c|}
\hline \multirow{3}{*}{$N_{1}=320$} & anglais & français & espagnol & allemand & russe & italien & autre \\
\cline { 2 - 7 } & 317 & 60 & 16 & 42 & 15 & 3 & 8 \\
& $(99 \%)$ & $(19 \%)$ & $(5 \%)$ & $(13 \%)$ & $(5 \%)$ & $(1 \%)$ & $(3 \%)$ \\
\hline
\end{tabular}

Tableau 5 : Les apprenants en FLE : les langues apprises à l'école primaire. 
Les représentations de la langue française chez des élèves polonophones...

\begin{tabular}{|c|c|c|c|c|c|c|c|}
\hline \multirow{2}{*}{$\mathrm{N}_{1}=320$} & anglais & français & espagnol & allemand & russe & italien & autre \\
\cline { 2 - 7 } & 307 & 168 & 50 & 104 & 14 & 2 & 12 \\
& $(96 \%)$ & $(53 \%)$ & $(16 \%)$ & $(33 \%)$ & $(4 \%)$ & $(1 \%)$ & $(4 \%)$ \\
\hline
\end{tabular}

Tableau 6 : Les apprenants en FLE : les langues apprises au collège.

II s'ensuit que, parmi les apprenants en FLE $\left(\mathrm{N}_{1}=320\right)$, ce sont les femmes qui dominent. $57 \%$ des apprenants suivent la première année d'apprentissage au lycée (1617 ans), 33 \% des enquêtés sont en deuxième classe (17-18 ans) et seulement $10 \%$ fréquentent la classe terminale (18-19 ans). La moitié des élèves apprend le français à raison de 3 heures par semaine. Néanmoins, les élèves qui ont 5 heures ou plus de cours de français par semaine sont assez nombreux (39\%), ce qui résulte probablement du fait qu'une partie de la recherche a été réalisée dans un établissement bilingue. En ce qui concerne la biographie langagière, elle est assez variée. Certes, presque toutes les personnes interrogées ont suivi les cours d'anglais aussi bien à l'école primaire qu'au collège, mais une grande partie des élèves a appris le français à l'école primaire ( $19 \%$ ) et au collège (53\%), ce qui peut avoir un impact sur le choix du français au lycée, car nous pouvons supposer qu'ils souhaitent continuer l'apprentissage de la langue à l'étape suivante de leur parcours scolaire.

\section{Les motifs de l'apprentissage du français}

Les apprenants en FLE ont été également questionnés sur les motifs qui les avaient poussés à apprendre le français au lycée. Le tableau qui suit présente leurs réponses de façon synthétique :

\begin{tabular}{|c|l|c|c|}
\hline $\mathbf{N}^{\circ}$ & les motifs $\left(\mathbf{N}_{\mathbf{1}}=\mathbf{3 2 0}\right)$ & OUI & $\mathbf{N O N}$ \\
\hline 1. & apprentissage imposé par l'école & $\begin{array}{c}148 \\
(46 \%)\end{array}$ & $\begin{array}{c}172 \\
(54 \%)\end{array}$ \\
\hline 2. & choix autonome de l'apprenant & $\begin{array}{c}186 \\
(58 \%)\end{array}$ & $\begin{array}{c}134 \\
(42 \%)\end{array}$ \\
\hline 3. & choix influencé par les parents de l'apprenant & $\begin{array}{c}56 \\
(18 \%)\end{array}$ & $\begin{array}{c}264 \\
(82 \%)\end{array}$ \\
\hline 4. & volonté d'étudier dans un pays francophone & $\begin{array}{c}118 \\
(37 \%)\end{array}$ & $\begin{array}{c}202 \\
(63 \%)\end{array}$ \\
\hline 5. & atout supplémentaire sur le marché du travail & $\begin{array}{c}250 \\
(78 \%)\end{array}$ & $\begin{array}{c}70 \\
(22 \%)\end{array}$ \\
\hline
\end{tabular}

Tableau 7 : Les motifs de l'apprentissage du français.

En analysant les réponses des apprenants en FLE $\left(\mathrm{N}_{1}=320\right)$, il est intéressant de constater que, dans la majorité des cas, la décision de commencer ou de continuer l'apprentissage était une décision autonome des apprenants interrogés (58\%). Néanmoins, nombreux sont aussi les élèves à qui l'école a imposé l'apprentissage de la langue (46\%). Un groupe d'élèves relativement important (37 
\%) apprend le français parce qu'il veut étudier dans un pays francophone. II faut également souligner que $78 \%$ des interrogés apprennent le français pour être concurrentiels sur le marché du travail. Les réponses aux deux derniers items témoignent du fait que les apprenants ont choisi le FLE pour son utilité présumée.

Dans l'une des questions de l'enquête, nous avons demandé aux apprenants s'ils auraient choisi l'apprentissage du français au lycée s'ils avaient dû choisir encore une fois l'apprentissage des langues. Le tableau ci-dessous illustre leurs réponses:

\begin{tabular}{|c|c|c|c|c|c|c|}
\hline & $\begin{array}{c}\text { décidément } \\
\text { oui }\end{array}$ & $\begin{array}{c}\text { plutôt } \\
\text { oui }\end{array}$ & $\begin{array}{c}\text { ni oui } \\
\text { ni non }\end{array}$ & $\begin{array}{c}\text { plutôt } \\
\text { non }\end{array}$ & $\begin{array}{c}\text { décidément } \\
\text { non }\end{array}$ & $\begin{array}{c}\text { manque } \\
\text { d'informations }\end{array}$ \\
\hline $\mathbf{N}_{\mathbf{1}}=\mathbf{3 2 0}$ & 151 & 90 & 26 & 34 & 16 & 1 \\
\hline$\%$ & $47 \%$ & $28 \%$ & $8 \%$ & $11 \%$ & $5 \%$ & $1 \%$ \\
\hline
\end{tabular}

Tableau 8 : Le choix hypothétique du français.

Les données recueillies montrent que les trois quarts des apprenants auraient choisi d'étudier le français s'ils avaient été confrontés à la prise d'une telle décision encore une fois (les réponses " décidément oui » et " plutôt oui " prises ensemble). Les élèves qui auraient choisi une autre langue sont minoritaires (les réponses " plutôt non » et " décidément non » prises ensemble). On peut donc supposer que la majorité des interrogés maintiennent leur motivation initiale pour l'apprentissage du français, probablement pour les raisons mentionnées plus haut.

\section{Les expériences relatives à l'apprentissage du français}

La question suivante de l'enquête portait sur les expériences des apprenants relatives à l'apprentissage. En d'autres termes, nous avons demandé aux élèves d'estimer la facilité du développement de différentes compétences en langue française (compréhension de l'oral, compréhension des écrits, production et interaction orales, production écrite), de même que la facilité de l'apprentissage de systèmes langagiers (grammaire et phonétique). Pour chacune des catégories, les personnes interrogées ont attribué une valeur de 1 à $5^{6}$, ce qui nous a permis de compter la moyenne arithmétique suivie de l'écart type. L'analyse des réponses des apprenants est présentée dans le tableau ci-dessous :

\footnotetext{
${ }^{6} 1$ =très difficile ; 2 =difficile ; $3=$ ni facile ni difficile ; $4=$ facile ; 5 = très facile
} 
Les représentations de la langue française chez des élèves polonophones...

\begin{tabular}{|l|l|c|c|}
\hline & & \multicolumn{2}{|c|}{$\mathbf{N}_{\mathbf{1}}=\mathbf{3 2 0}$} \\
\hline $\mathbf{N}^{\circ}$ & & $\begin{array}{c}\text { moyenne } \\
\text { (sur 5) }\end{array}$ & $\begin{array}{c}\text { écart } \\
\text { type }\end{array}$ \\
\hline 1. & compréhension de l'oral & 2,8 & 1,2 \\
\hline 2. & compréhension des écrits & 3,6 & 0,9 \\
\hline 3. & production/interaction orale & 3,3 & 2,6 \\
\hline 4. & production écrite & 3,2 & 1,1 \\
\hline 5. & grammaire & 3,0 & 1,2 \\
\hline 6. & prononciation & 3,4 & 1,2 \\
\hline
\end{tabular}

Tableau 9 : Estimation de la facilité de l'apprentissage du français.

Les données recueillies montrent que - en général - la langue française ne passe pas pour une langue facile chez les apprenants, car la moyenne la plus élevée ne dépasse pas 3,6. En analysant les résultats, nous pouvons constater que c'est la compréhension des écrits et la prononciation qui posent le moins de difficultés aux apprenants. C'est aussi le cas de la production/interaction orale, sauf que pour cet item - l'écart type est relativement élevé, ce qui prouve la dispersion des réponses et ne nous autorise pas à généraliser les résultats. La production écrite n'est pas non plus trop problématique pour les élèves. La compétence la plus difficile, c'est la compréhension de l'oral. Cet état de choses peut s'expliquer par le fait que la langue française est assez éloignée de la langue polonaise, la première langue des apprenants, mais aussi par le fait que les élèves sont rarement exposés à cette langue en dehors de la salle de classe.

\section{Le contact avec le français}

Nous avons également intégré dans le questionnaire de l'enquête des questions concernant les séjours des élèves dans des pays francophones et la connaissance du français par des personnes appartenant à l'environnement immédiat de l'apprenant. Les données recueillies sont présentées dans le tableau qui suit :

\begin{tabular}{|c|l|c|c|}
\hline \multirow{2}{*}{$\mathbf{N}^{\circ}$} & & \multicolumn{2}{|c|}{$\mathbf{N}_{\mathbf{1}}=\mathbf{3 2 0}$} \\
\cline { 3 - 4 } & & OUI & NON \\
\hline 1. & au moins UN séjour en France & $\begin{array}{c}210 \\
(66 \%)\end{array}$ & $\begin{array}{c}110 \\
(34 \%)\end{array}$ \\
\hline 2. & $\begin{array}{l}\text { au moins UN séjour dans un pays francophone autre que la } \\
\text { France }\end{array}$ & $\begin{array}{c}141 \\
(44 \%)\end{array}$ & $\begin{array}{c}179 \\
(56 \%)\end{array}$ \\
\hline 3. & connaissance du français par les cohabitants de l'apprenant & $\begin{array}{c}92 \\
(29 \%)\end{array}$ & $\begin{array}{c}228 \\
(71 \%)\end{array}$ \\
\hline 4. & connaissance du français par les amis de l'apprenant & $\begin{array}{c}276 \\
(86 \%)\end{array}$ & $\begin{array}{c}44 \\
(14 \%)\end{array}$ \\
\hline 5. & $\begin{array}{l}\text { présence des personnes francophones dans le quartier de l'ap- } \\
\text { prenant }\end{array}$ & $\begin{array}{c}80 \\
(25 \%)\end{array}$ & $\begin{array}{c}240 \\
(75 \%)\end{array}$ \\
\hline
\end{tabular}

Tableau 10 : Les apprenants en FLE : le contact avec le français en dehors de l'école. 
Il est intéressant de constater que $86 \%$ des élèves interrogés déclarent avoir des amis qui connaissent le français. $66 \%$ des personnes interrogées ont séjourné au moins une fois en France, contre $44 \%$ qui déclarent au moins un séjour dans au pays francophone autre que la France. Néanmoins, rares sont les élèves dont les cohabitants connaissent le français ( $29 \%$ ), de même que les apprenants qui rencontrent des francophones dans leur environnement immédiat ( $25 \%$ ). Cela reste conforme au caractère monolingue de la Pologne que nous avons brièvement évoqué au début du présent article et qui influence significativement le processus d'enseignement/apprentissage des langues à l'école polonaise. Force est quand même de constater que les élèves qui partent dans un pays francophone et dont les amis connaissent le français sont assez nombreux, ce qui peut avoir une influence positive sur la motivation pour l'apprentissage de la langue en question. De plus, cela nous permet également de supposer que les séjours dans les pays francophones - même d'une courte durée - et les amis/camardes connaissant le français peuvent également influer sur la perception du français par les élèves interrogés. Cependant, nous ne pouvons pas ignorer le fait que les mêmes facteurs peuvent également être la conséquence de l'apprentissage du français.

\subsubsection{Les non-apprenants en FLE}

\section{La caractéristique générale}

En ce qui concerne les non-apprenants en FLE, ils sont moins nombreux dans notre échantillon $\mathrm{N}_{2}=236$. Dans les tableaux qui suivent, nous présentons leur caractéristique générale.

\begin{tabular}{|c|c|c|c|}
\hline \multirow{2}{*}{$N_{2}=236$} & femme & homme & $\begin{array}{c}\text { manque } \\
\text { d'informations }\end{array}$ \\
\cline { 2 - 4 } & $\begin{array}{c}126 \\
(53 \%)\end{array}$ & $\begin{array}{c}108 \\
(46 \%)\end{array}$ & $\begin{array}{c}2 \\
(1 \%)\end{array}$ \\
\hline
\end{tabular}

Tableau 11 : Les non-apprenants en FLE : sexe.

\begin{tabular}{|c|c|c|c|c|}
\hline \multirow{2}{*}{$N_{2}=236$} & $\mathbf{1}^{\text {ì̀re }}$ & $\mathbf{2}^{\mathbf{e}}$ & $\mathbf{3}^{\mathbf{e}}$ (terminale) & $\begin{array}{c}\text { manque } \\
\text { d'informations }\end{array}$ \\
\cline { 2 - 5 } & $\begin{array}{c}45 \\
(19 \%)\end{array}$ & $\begin{array}{c}112 \\
(47 \%)\end{array}$ & $\begin{array}{c}78 \\
(33 \%)\end{array}$ & $\begin{array}{c}1 \\
(1 \%)\end{array}$ \\
\hline
\end{tabular}

Tableau 12 : Les non-apprenants en FLE : classe.

\begin{tabular}{|c|c|c|c|c|c|c|c|}
\hline \multirow{3}{*}{$\mathrm{N}_{2}=236$} & anglais & français & espagnol & allemand & russe & italien & autre \\
\cline { 2 - 8 } & 226 & 11 & 13 & 79 & 22 & 1 & 2 \\
& $(96 \%)$ & $(5 \%)$ & $(6 \%)$ & $(33 \%)$ & $(9 \%)$ & $(1 \%)$ & $(1 \%)$ \\
\hline
\end{tabular}

Tableau 13 : Les non-apprenants en FLE : les langues apprises à l'école primaire. 
Les représentations de la langue française chez des élèves polonophones...

\begin{tabular}{|c|c|c|c|c|c|c|c|}
\hline \multirow{3}{*}{$\mathrm{N}_{2}=236$} & anglais & français & espagnol & allemand & russe & italien & autre \\
\cline { 2 - 7 } & 225 & 10 & 44 & 175 & 17 & 0 & 6 \\
& $(96 \%)$ & $(4 \%)$ & $(19 \%)$ & $(74 \%)$ & $(7 \%)$ & $(0 \%)$ & $(3 \%)$ \\
\hline
\end{tabular}

Tableau 14 : Les non-apprenants en FLE : les langues apprises au collège.

Il résulte des tableaux présentés ci-dessus que la proportion entre le nombre des femmes et des hommes est plus équilibrée que par rapport aux apprenants en FLE : $53 \%$ de femmes contre $46 \%$ d'hommes participent à l'étude. La majorité des élèves interrogés suit la deuxième année d'apprentissage au lycée : ils ont donc entre 17 et 18 ans. Leur biographie langagière est également variée. Presque tous les élèves ont suivi les cours d'anglais à l'école primaire et au collège, mais $75 \%$ des enquêtés ont étudié l'allemand au collège (contre $33 \%$ au primaire). Les élèves qui ont suivi les cours de français à l'école primaire et au collège sont peu nombreux : $5 \%$ pour l'école primaire et $4 \%$ pour le collège. La question qui se pose alors est de savoir quelles ont été les raisons de l'abandon de l'apprentissage de la langue en question.

\section{Les motifs du non-apprentissage}

On a demandé aux non-apprenants en FLE pourquoi ils n'avaient pas décidé d'apprendre le français au lycée. Le tableau ci-dessous présente leurs réponses :

\begin{tabular}{|c|l|c|c|}
\hline $\mathbf{N}^{\circ}$ & \multicolumn{1}{|c|}{ les motifs ( $\left.\mathbf{N}_{\mathbf{2}}=\mathbf{2 3 6}\right)$} & OUI & NON \\
\hline 1. & $\begin{array}{l}\text { l'établissement ne propose pas l'apprentissage du fran- } \\
\text { çais au niveau correspondant aux besoins de l'apprenant }\end{array}$ & $\begin{array}{c}12 \\
(5 \%)\end{array}$ & $\begin{array}{c}224 \\
(95 \%)\end{array}$ \\
\hline 2. & $\begin{array}{l}\text { l'établissement ne propose pas du tout l'apprentissage du } \\
\text { français }\end{array}$ & $\begin{array}{c}4 \\
(2 \%)\end{array}$ & $\begin{array}{c}232 \\
(98 \%)\end{array}$ \\
\hline 3. & la langue française ne plaisait pas à l'apprenant & $\begin{array}{c}120 \\
(51 \%)\end{array}$ & $\begin{array}{c}116 \\
(49 \%)\end{array}$ \\
\hline 4. & la langue française semblait difficile à l'apprenant & $\begin{array}{c}126 \\
(54 \%)\end{array}$ & $\begin{array}{c}110 \\
(46 \%)\end{array}$ \\
\hline 5. & $\begin{array}{l}\text { l'apprenant n'a pas voulu continuer l'apprentissage du } \\
\text { français }\end{array}$ & $\begin{array}{c}30 \\
(13 \%)\end{array}$ & $\begin{array}{c}206 \\
(87 \%)\end{array}$ \\
\hline 6. & l'apprenant ne trouvait pas le français utile pour l'avenir & $\begin{array}{c}147 \\
(62 \%)\end{array}$ & $\begin{array}{c}89 \\
(38 \%)\end{array}$ \\
\hline
\end{tabular}

Tableau 15 : Les motifs du non-apprentissage.

L'analyse des données recueilles nous permet de constater les trois raisons majeures de la décision des apprenants : ils ne trouvaient pas la langue française utile pour l'avenir (62\%), le français leur semblait être une langue difficile à apprendre (54\%) et le français ne leur plaisait pas (51\%). II nous semble nécessaire de remarquer que les raisons énumérées peuvent probablement avoir aussi un impact sur les représentations des apprenants relatives au français 
et à son utilité. Il est aussi intéressant de constater que $13 \%$ des élèves ont pris la décision de ne pas continuer l'apprentissage, ce qui peut peut-être se traduire par différentes causes : mauvais résultats scolaires, difficultés dans l'apprentissage résultant de la spécificité de la langue française ou autres. Force est également de constater que seulement $5 \%$ des interrogés n'ont pas eu la possibilité d'apprendre le français, car il était absent de l'offre éducative de l'établissement choisi par l'apprenant.

\section{Le contact avec le français}

Les non-apprenants en FLE ont été également questionnés sur leurs séjours dans des pays francophones et la connaissance du français par les gens de leur environnement immédiat. L'objectif de cet ensemble de questions était de diagnostiquer l'intensité du contact avec le français et la culture française au sens large du terme, car cela peut influencer les représentations des élèves relatives à la langue française. Le tableau suivant présente les réponses des apprenants interrogés :

\begin{tabular}{|c|l|c|c|}
\hline \multirow{2}{*}{$\mathbf{N}^{\circ}$} & \multicolumn{2}{|c|}{$\mathbf{N}_{\mathbf{2}}=\mathbf{2 3 6}$} \\
\cline { 3 - 4 } & & OUI & NON \\
\hline 1. & au moins UN séjour en France & $\begin{array}{c}100 \\
(42 \%)\end{array}$ & $\begin{array}{c}136 \\
(58 \%)\end{array}$ \\
\hline 2. & $\begin{array}{l}64 \\
\text { France }\end{array}$ & $\begin{array}{c}172 \\
(73 \%)\end{array}$ \\
\hline 3. & connaissance du français par les cohabitants de l'apprenant & $\begin{array}{c}48 \\
(20 \%)\end{array}$ & $\begin{array}{c}188 \\
(80 \%)\end{array}$ \\
\hline 4. & connaissance du français par les amis de l'apprenant & $\begin{array}{c}186 \\
(79 \%)\end{array}$ & $\begin{array}{c}50 \\
(21 \%)\end{array}$ \\
\hline 5. & $\begin{array}{l}\text { présence des personnes francophones dans le quartier de } \\
\text { l'apprenant }\end{array}$ & $\begin{array}{c}40 \\
(17 \%)\end{array}$ & $\begin{array}{c}196 \\
(83 \%)\end{array}$ \\
\hline
\end{tabular}

Tableau 16 : Les non-apprenants en FLE : le contact avec le français en dehors de l'école.

En analysant les réponses des apprenants interrogés, nous constatons qu'elles diffèrent de façon significative des réponses données par les apprenants en FLE. Seuls $42 \%$ des enquêtés ont séjourné au moins une fois en France contre $27 \%$ qui sont partis au moins une fois dans un pays francophone. Les personnes francophones sont rarement présentes dans l'environnement immédiat des élèves (17\%). Seulement $20 \%$ des interrogés déclarent avoir des cohabitants qui parlent français, mais $79 \%$ des apprenants ont des amis connaissant la langue en question. On peut donc supposer que le contact quotidien avec la langue française reste assez limité, ce qui influence les représentations des élèves relatives à cette langue. 
Pour faire le point, il convient de constater que les différences majeures dans les caractéristiques générales du public apprenant le FLE et celui ayant choisi une autre langue étrangère au lycée, concernent la perception de la difficulté, de la beauté et de l'utilité du français. Quant aux séjours dans les pays francophones ou aux contacts avec les natifs, si l'on constate une plus forte intensité de ses contacts chez les apprenants en FLE, ceci peut être la raison mais aussi bien la conséquence du choix de l'apprentissage de la langue française - malheureusement, la formulation de la question ne nous permet pas de trancher sur la direction de la relation de cause à effet.

Dans la partie suivante, nous passons à l'analyse des représentations du FLE chez les apprenants en FLE et les non-apprenants en FLE qui se laissent déchiffrer dans la suite du questionnaire.

\section{La discussion des résultats}

\subsection{Les représentations des élèves relatives à la langue française}

Pour examiner les représentations des apprenants relatives à la langue française, nous leur avons proposé 26 items par rapport auxquels ils devaient se positionner en choisissant une des valeurs de 1 à $5^{7}$, ce qui nous a permis de compter la moyenne arithmétique suivie de l'écart type. Les items se référaient à 5 domaines d'analyse :

- le français en tant que phénomène linguistique ;

- le français en tant que phénomène esthétique ;

- le français en tant que phénomène culturel ;

- le français en tant que phénomène social ;

- le français en tant que phénomène politique.

Dans les parties qui suivent, nous nous référons aux catégories énumérées ci-dessus.

\subsubsection{Le français en tant que phénomène linguistique}

La langue française passe souvent - pour les Polonais - pour une langue difficile, à cause d'une prononciation spécifique et d'une grammaire trop compliquée. Les polonophones remarquent aussi que les Français parlent trop vite, ce qui les empêche de comprendre les locuteurs natifs parlant français. Pour cette raison, nous avons intégré les items concernant la spécificité de la langue française dans notre questionnaire de l'enquête. Le tableau ci-dessous montre les réponses des élèves :

${ }^{7}$ 1=décidément non, 2=plutôt non, 3=je ne sais pas, 4=plutôt oui, 5=décidément oui. 
Radosław Kucharczyk, Krystyna Szymankiewicz

\begin{tabular}{|c|l|c|c|c|c|}
\hline $\mathbf{N}^{\circ}$ & La langue française me fait penser à/au ... & \multicolumn{2}{|c|}{$\mathbf{N}_{\mathbf{1}}=\mathbf{3 2 0}$} & \multicolumn{2}{c|}{$\mathbf{N}_{\mathbf{2}}=\mathbf{2 3 6}$} \\
\hline & & moyenne & écart type & moyenne écart type \\
\hline 1. & une prononciation difficile & 3,7 & 1,5 & 4,1 & 1,1 \\
\hline 2. & une grammaire logique & 2.7 & 1.6 & 2,4 & 0,9 \\
\hline 3. & une articulation spécifique de [R] & 4,7 & 1,2 & 4,1 & 1,0 \\
\hline 4. & un débit rapide & 4.1 & 1.5 & 3,4 & 2,9 \\
\hline
\end{tabular}

Tableau 17 : La langue française en tant que phénomène linguistique.

En analysant les réponses des apprenants interrogés, nous pouvons constater que la prononciation française semble être assez difficile aussi bien pour $N_{1}$ que pour $\mathrm{N}_{2}$. Les deux groupes associent le français à une articulation spécifique de $[R]$. Quand au débit, il est plus présent dans les représentations de $N_{1}$ que dans celles de $N_{2}$, ce qui peut résulter de l'expérience du premier groupe. Force est quand même de constater que l'écart type est assez élevé, ce qui prouve la dispersion des réponses choisies par les apprenants. Quant à la grammaire française, les deux groupes la trouvent moyennement logique, mais l'écart type est plus élevé pour $N_{1}$. Tout compte fait, il n'y a pas de grandes différences entre les représentations de la langue française en tant que phénomène linguistique chez les apprenants en FLE et celles exprimées par les non-apprenants en FLE.

\subsubsection{Le français en tant que phénomène esthétique}

Une partie des items inclus dans notre cafétéria se référait aux dimensions esthétiques de la langue française. En effet, le français est souvent associé à la langue des élites, ce qui résulte probablement de la place qu'il occupait en Pologne au $19^{\mathrm{e}}$ siècle. Il fait aussi penser à l'amour (Paris, la ville des amoureux). De plus, il se caractérise également par une sonorité agréable. Le tableau ci-dessous montre les réponses des apprenants :

\begin{tabular}{|c|l|c|c|c|c|}
\hline $\mathbf{N}^{\mathbf{2}}$ & La langue française me fait penser à/au ... & \multicolumn{2}{|c|}{$\mathbf{N}_{\mathbf{1}}=\mathbf{3 2 0}$} & \multicolumn{2}{c|}{$\mathbf{N}_{\mathbf{2}}=\mathbf{2 3 6}$} \\
\hline & & moyenne & écart type & moyenne & écart type \\
\hline 1. & l'élégance & 4,3 & 0,9 & 3,9 & 1,0 \\
\hline 2. & un esprit romantique & 4,1 & 1,0 & 3,7 & 1,2 \\
\hline 3. & un caractère exceptionnel & 3,4 & 1,3 & 2,8 & 1,3 \\
\hline 4. & prestige & 3,5 & 1,3 & 2,7 & 1,3 \\
\hline 5. & une langue élitiste & 3,4 & 1,5 & 2,6 & 1,4 \\
\hline 6. & une sonorité agréable & 4,4 & 1,3 & 3,4 & 1,3 \\
\hline 7. & une sonorité peu masculine & 2,5 & 1,7 & 2,9 & 1,2 \\
\hline
\end{tabular}

Tableau 18 : La langue française en tant que phénomène esthétique. 
L'analyse des réponses des deux groupes d'élèves montre qu'ils associent la langue française surtout à l'élégance et à un esprit romantique, mais les moyennes sont légèrement plus élevées pour $N_{1}$. Les différences dans les moyennes sont plus visibles dans l'analyse des autres items se référant aux caractéristiques de la langue françaises telles que : caractère exceptionnel, prestige, langue élitiste et sonorité agréable. Même si l'écart type reste toujours élevé, les moyennes sont plus hautes pour $\mathrm{N}_{1}$ ce qui, à nos yeux, confirme encore une fois leur attitude positive pour le français qui s'est maintenue tout au long de l'apprentissage.

\subsubsection{Le français en tant que phénomène culturel}

Une partie de notre questionnaire d'enquête portait sur les aspects culturels que la langue française peut véhiculer. En effet, le français peut être associé soit à des éléments faisant partie de la culture basse (cuisine, mode), de la culture populaire (chanson) ou de la culture cultivée (art, littérature, film, événements historiques). Les réponses des élèves interrogés sont présentées dans le tableau qui suit :

\begin{tabular}{|c|l|c|c|c|c|}
\hline $\mathbf{N}^{\mathbf{2}}$ & La langue française me fait penser à/au ... & \multicolumn{2}{|c|}{$\mathbf{N}_{\mathbf{1}}=\mathbf{3 2 0}$} & \multicolumn{2}{c|}{$\mathbf{N}_{\mathbf{2}}=\mathbf{2 3 6}$} \\
\hline & & moyenne & écart type & moyenne & écart type \\
\hline 1. & une cuisine délicieuse & 3,6 & 1,1 & 3,3 & 1,1 \\
\hline 2. & la mode & 4,2 & 1,0 & 3,9 & 1,0 \\
\hline 3. & I'art & 4,0 & 1,0 & 3,6 & 1,1 \\
\hline 4. & la littérature & 3,5 & 1,1 & 3,1 & 1,2 \\
\hline 5. & film français & 3,6 & 1,3 & 3,0 & 1,3 \\
\hline 6. & des événements historiques importants & 3,4 & 1,2 & 3,1 & 1,2 \\
\hline 7. & la chanson française & 3,7 & 1,3 & 3,0 & 1,3 \\
\hline
\end{tabular}

Tableau 19 : La langue française en tant que phénomène culturel.

Pareillement aux réponses des élèves analysées plus haut, les moyennes pour $N_{1}$ sont plus élevées que celles pour $\mathrm{N}_{2}$, ce qui peut s'expliquer encore une fois par une meilleure connaissance de la culture française et francophone dans le sens large du terme, résultant de l'apprentissage du français à l'école. La langue française est surtout associée au monde de la mode, à la chanson française et puis à la tradition culinaire. Viennent ensuite des éléments tels que les films français, la littérature et des événements historiques importants. Dans les deux groupes, les moyennes sont relativement élevées (elles ne sont jamais plus basses que 3,0 ) et l'écart type n'est pas trop haut, ce qui nous permet de supposer que l'image de la langue française en tant que langue qui transmet certaines valeurs culturelles françaises et francophones, est présente dans la pensée des apprenants. 


\subsubsection{Le français en tant que phénomène social}

L'une des caractéristiques de chacune des langues est sa fonction sociale, car chaque langue est un outil privilégié de l'interaction humaine. Sans nul doute, $c^{\prime}$ est aussi le cas du français qui est la $5^{\mathrm{e}}$ langue la plus parlée au monde (300 millions de locuteurs) après le chinois, l'anglais, l'espagnol et l'arabe (Semo, 2018). Nous avons donc demandé aux apprenants participant à l'étude s'ils associaient la langue française au multiculturalisme (Francophonie), à des personnes et voyages intéressants ou à des endroits intéressants à visiter. Le tableau ci-dessous montre leurs réponses :

\begin{tabular}{|c|l|c|c|c|c|}
\hline $\mathbf{N}^{\circ}$ & La langue française me fait penser à/au ... & \multicolumn{2}{|c|}{$\mathbf{N}_{\mathbf{1}}=\mathbf{3 2 0}$} & \multicolumn{2}{c|}{$\mathbf{N}_{\mathbf{2}}=\mathbf{2 3 6}$} \\
\hline & & moyenne & écart type & moyenne & écart type \\
\hline 1. & multiculturalisme & 3,7 & 1,3 & 3,4 & 1,3 \\
\hline 2. & des personnes intéressantes & 3,3 & 1,3 & 2,9 & 1,1 \\
\hline 3. & des voyages intéressants & 3,9 & 1,2 & 3,4 & 1,1 \\
\hline 4. & des endroits intéressants dans le monde & 4,0 & 1,2 & 3,5 & 1,1 \\
\hline
\end{tabular}

Tableau 20 : La langue française en tant que phénomène social.

Comme dans des analyses précédentes, les moyennes pour $\mathrm{N}_{1}$ sont légèrement plus élevées que celles de $\mathrm{N}_{2}$, ce qui résulte probablement de leur participation aux cours de FLE et du contact plus intense avec le français en dehors de la salle de classe. Les élèves interrogés associent surtout le français à des endroits intéressants dans le monde, mais aussi au multiculturalisme. Les moyennes concernant les voyages sont aussi hautes, ce qui n'est pas le cas pour les rencontres avec des personnes intéressantes. Dans les deux groupes, l'écart type n'est pas élevé, donc les réponses fournies par les élèves ne sont pas trop dispersées.

\subsubsection{Le français en tant que phénomène politique}

Le dernier bloc d'items relatif aux représentations que les apprenants se font de la langue française se référait à la dimension politique de la langue. En effet, le français est l'une des langues officielles de l'Union européenne, et par voie de conséquence, il est une langue souvent utilisée dans les échanges diplomatiques. On peut donc supposer qu'il est également une langue de communication internationale, vu son nombre de locuteurs que nous avons mentionné plus haut. Finalement, pour des raisons historiques (la Révolution de 1789), le français est souvent associé aux valeurs démocratiques, telles que la liberté, l'égalité et la fraternité. Ces valeurs démocratiques se reflètent également 
Les représentations de la langue française chez des élèves polonophones...

dans la position de la France au sein de l'Union européenne. Le tableau cidessous présente les réponses des apprenants interrogés :

\begin{tabular}{|c|l|c|c|c|c|}
\hline $\mathbf{N}^{\circ}$ & La langue française me fait penser à ... & \multicolumn{2}{c|}{$\mathbf{N}_{\mathbf{1}}=\mathbf{3 2 0}$} & \multicolumn{2}{c|}{$\mathbf{N}_{\mathbf{2}}=\mathbf{2 3 6}$} \\
\hline & & moyenne & écart type & moyenne & écart type \\
\hline 1. & la diplomatie & 3,0 & 1,2 & 2,8 & 1,2 \\
\hline 2. & une langue de communication internationale & 2,9 & 1,5 & 2,2 & 1,1 \\
\hline 3. & des institutions de I'Union européenne & 2,9 & 1,7 & 2,8 & 1,2 \\
\hline 4. & des valeurs démocratiques & 2,8 & 1,7 & 2,6 & 1,2 \\
\hline
\end{tabular}

Tableau 21 : La langue française en tant que phénomène politique.

L'analyse des réponses des deux groupes d'apprenants participant à l'étude montre qu'il n'existe pas de différences significatives entre $N_{1}$ et $N_{2}$, ce qui semble étonnant pour les apprenants en FLE qui devraient associer la langue française suite à l'apprentissage scolaire - aux institutions de l'Union européenne et à des valeurs démocratiques. En général, les apprenants interrogés perçoivent avant tout le français en tant que langue de la diplomatie, plus rarement en tant que langue de communication internationale ou langue qui véhicule des valeurs démocratiques. Il faut également noter que l'écart type n'est pas élevé dans les deux groupes, ce qui prouve encore une fois une basse dispersion des réponses choisies.

\subsection{Les représentations des élèves relatives à l'utilité de la langue française}

Le dernier bloc de questions dans notre questionnaire d'enquête avait pour objectif d'examiner les représentations des élèves concernant l'utilité de la langue française. Pour ce faire, nous avons listé différentes situations de la vie quotidienne durant desquelles la langue française peut s'avérer utile. Nous les présentons dans le tableau qui suit, accompagnées de l'analyse des réponses des apprenants interrogés :

\begin{tabular}{|c|l|c|c|c|c|}
\hline & \multicolumn{2}{|c|}{$\mathbf{N}_{\mathbf{1}}=\mathbf{3 2 0}$} & \multicolumn{2}{c|}{$\mathbf{N}_{\mathbf{2}}=\mathbf{2 3 6}$} \\
\hline $\mathbf{N}^{\circ}$ & \multicolumn{1}{|c|}{ Le français peut être utile pour... } & moyenne & écart type & moyenne & écart type \\
\hline 1. & les voyages & 3,2 & 1,3 & 2,4 & 0,8 \\
\hline 2. & la vie personnelle & 2,3 & 0,9 & 1,6 & 0,7 \\
\hline 3. & les études & 3,1 & 0,9 & 1,9 & 0,8 \\
\hline 4. & la future vie professionnelle & 3,1 & 0,8 & 2,2 & 0,9 \\
\hline 5. & trouver un bon emploi & 3,4 & 0,8 & 2,4 & 1,0 \\
\hline 6. & les contacts avec les étrangers & 3,4 & 0,7 & 2,7 & 0,9 \\
\hline 7. & sa propre satisfaction & 3,4 & 0,8 & 2,6 & 1,1 \\
\hline 8. & utiliser les nouvelles technologies & 1,7 & 0,8 & 1,3 & 0,6 \\
\hline 9. & lire des livres, la presse, etc. & 2,6 & 1,0 & 1,9 & 0,8 \\
\hline 10. & se distraire (films, télévision, musique, jeux) & 2,8 & 0,9 & 1,9 & 0,9 \\
\hline
\end{tabular}

Tableau 22 : L'utilité de la langue française. 
L'analyse des réponses des élèves montre qu'ils perçoivent la langue française moyennement utile dans la vie quotidienne : néanmoins, les apprenants du groupe $\mathrm{N} 1$ voient plus d'avantages découlant de sa connaissance que leurs homologues du groupe N2, ce qui reste en corrélation, d'après nous, avec leur motivation primaire pour commencer l'apprentissage du français. Ils le trouvent utile pour des raisons professionnelles ou éducatives (trouver un « bon " travail, travail et apprentissage ultérieurs), mais aussi personnelles (communiquer avec des étrangers, satisfaction personnelle, voyages). La langue française est quand même moins utile pour se divertir (lire en français, regarder des films, écouter de la musique). De plus les élèves ne voient pas l'intérêt de recourir au français lors de l'usage de l'ordinateur ou d'autres appareils techniques, ce qui n'est pas étonnant puisque c'est l'anglais qui est la langue dominante dans le domaine des nouvelles technologies.

\section{Conclusions}

Au terme de notre analyse, il convient de formuler des réponses à nos questions de recherche et, ainsi, de confirmer ou d'infirmer nos hypothèses. Premièrement, les représentations relatives à la langue française fonctionnant chez les jeunes Polonais se sont avérées, comme nous l'avions supposé, positives à l'égard du caractère culturel et esthétique de cette langue. En même temps, le français est perçu comme une langue plutôt difficile à apprendre par les polonophones et utile seulement dans quelques domaines de la vie, notamment dans le domaine professionnel.

Deuxièmement, si l'on compare le groupe d'apprenants en FLE avec celui de non-apprenants en FLE, force est de constater que l'image du français dans les deux groupes est semblable en ce qui concerne la perception de la langue en tant que phénomène linguistique, culturel et politique. Pourtant des différences se font remarquer quant aux critères esthétique et social : les apprenants en FLE trouvent le français plus beau et plus utile dans sa fonction d'outil de communication sociale que ceux qui ont renoncé à la possibilité d'apprendre le FLE. Bien évidemment, cette tendance peut être renforcée par le simple fait d'apprendre et d'utiliser le français mais, comme le prouvent les réponses aux questions $\mathrm{n}^{\circ}$ IV et VIII (voir le Tableau 1), elle a dû également motiver le choix de l'apprentissage du français par les répondants du premier groupe.

On peut en déduire que ce sont justement les critères esthétique, social et pragmatique qui semblent jouer un rôle positif dans le choix de l'apprentissage du français en tant que deuxième langue étrangère au lycée, ce qui vient confirmer notre hypothèse de départ. II est à noter que le contact avec les francophones dans l'entourage dont peuvent profiter certains jeunes Polonais y est pour beaucoup. 
Pour ce qui est des critères d'appréciation du français qui peuvent décourager les lycéens polonais de choisir l'apprentissage de cette langue en tant que deuxième langue étrangère, nous avons vu se confirmer notre hypothèse selon laquelle il s'agit ici surtout de la perception de la difficulté de la langue française accompagnée de la conviction que le français n'est pas très utile (ou aussi utile que l'anglais, ce qui est sous-entendu). Nous sommes persuadés que nombreux sont les élèves qui n'envisagent pas d'entreprendre l'apprentissage d'une langue perçue comme difficile, en sachant que son enseignement limité en nombre d'heures ne saura conduire qu'à une maîtrise insatisfaisante de cette langue.

Pour conclure, on peut constater que même si les jeunes Polonais possèdent une image assez complexe et plutôt positive de la langue française, leurs représentations ne contiennent pas, en règle générale, assez de composantes pragmatiques et affectives résultant du contact réel avec la langue qui pourraient faire pencher les lycéens vers l'apprentissage du français au lieu de celui de l'allemand ou celui du russe. Ce sont donc des facteurs personnels tels qu'une biographie langagière riche et des expériences interculturelles qui semblent apporter aux représentations du français la conviction de son utilité et la motivation interne à son apprentissage, ne serait-ce que pour le plaisir que l'usage de cette langue peut procurer.

\section{BIBLIOGRAPHIE}

Błaszkiewicz J. (2017), L'apprenant polonophone face à l'apprentissage d'une langue tertiaire : le français après l'anglais. (mémoire de maîtrise non publié, préparé sous la direction de Jolanta Sujecka-Zając). Varsovie : Université de Varsovie - Institut d'Études romanes.

Castellotti V. (2017), Pour une didactique de l'appropriation. Paris : Didier. Castellotti V., Moore D. (2002), Représentations sociales des langues et enseignements. Strasbourg : Conseil de l'Europe.

Chiffres clés de l'enseignement des langues à l'école en Europe. Rapport Eurydice.

(2017), Luxembourg : Office des publications de l'Union européenne.

Dabène L. (1997), L'image des langues et leur apprentissage (in :) Matthey M. (éds.), Les langues et leurs images. Neuchâtel : IRDP Editeur, 19-23.

Defays J.-M. (2018), Enseigner le français langue étrangère et seconde. Approche humaniste de la didactique des langues et des cultures. Bruxelles : MARDAGA Supérieur.

GUS (2018), Oświata i wychowanie w roku szkolnym 2017/2018, Warszawa/ Gdańsk : Główny Urząd Statystyczny/Urząd Statystyczny w Gdańsku. Jodelet D. (1993), Les représentations sociales. Regard sur la connaissance ordinaire. "Sciences humaines " $n^{\circ} 27,22-24$. 
Ministerstwo Edukacji Narodowej (2016), Wstęp do rozporzqdzenia Ministra Edukacji Narodowej z dnia 17 czerwca 2016 r. zmieniajqcego rozporzqdzenie w sprawie podstawy programowej wychowania przedszkolnego oraz kształcenia ogólnego w poszczególnych typach szkół. Online : https:// men.gov.pl/wp-content/uploads/2016/11/podstawa-programowa-\%E 2\%80\%93-jezyk-obcy.pdf [consulté 09.02.2018] ${ }^{8}$.

Moore D. (2001), Les représentations des langues et de leur apprentissage : itinéraires théoriques et trajets méthodologiques, (in :) Moore D. (éds.), Représentations des langues et de leur apprentissage. Références, modèles, données, méthodes. Paris : Didier, 7-22.

Moscovici S. (1961/1976), La psychanalyse, son image et son public. Paris : PUF.

Pudo D. (2016), Représentations du français et d'autres langues étrangères chez les étudiants de philologie romane. "Romanica Cracoviensia " $n^{\circ} 4$, 249-263. Online: http://www.ejournals.eu/Romanica-Cracoviensia/20 16/Tom-16-Numer-4/art/8846/ [consulté 15.04.2020]

Semo M. (2018), Le français, cinquième langue la plus parlée dans le monde. « Le monde Afrique » du 11 octobre 2018. Online : https://www.lemonde. fr/afrique/article/2018/10/11/le-francais-cinquieme-langue-la-plus-pa rlee-dans-le-monde_5367799_3212.html [consulté 16.06.2019]

Zarate G. (1993), Représentations de l'étranger et didactique des langues, Paris : Didier.

Ziółkowski M. (2004), La francophonie en Pologne. " Hermès, La Revue » n 40 (3), 59-61. Online: https://www.cairn.info/revue-hermes-la-revue-2004 -3-page-59.htm [consulté 15.04.2020].

8 „Wstęp" ne fait pas partie de „rozporządzenie”; ce document a été publié uniquement sur le site internet du Ministère de l'Education Nationale dans le cadre des consultations du projet du programme national (podstawa programowa). 